\title{
Correlation between galactic Hi and the Cosmic Microwave Background
}

\author{
Kate Land ${ }^{1}$ and Anže Slosar ${ }^{1,2}$ \\ ${ }^{1}$ Astrophysics, Denys Wilkinson Building, University of Oxford, Keble Road, OX3RH1, Oxford, UK and \\ ${ }^{2}$ Faculty of Mathematics and Physics, University of Ljubljana, Slovenia
}

(Dated: May 9, 2018)

\begin{abstract}
We revisit the issue of a correlation between the atomic hydrogen gas in our local Galaxy and the Cosmic Microwave Background (CMB), a detection of which has been claimed in some literature. We cross-correlate the 21-cm emission of Galactic atomic hydrogen as traced by the Leiden/Argentine/Bonn Galactic Hi survey with the 3-year CMB data from the Wilkinson Microwave Anisotropy Probe. We consider a number of angular scales, masks, and Hi velocity slices and find no statistically significant correlation.
\end{abstract}

PACS numbers:

\section{INTRODUCTION}

Since the beginning of this millenium significant advances in Cosmology have been made, in large part due to the advent of high-quality cosmological datasets. The full-sky results of the Cosmic Microwave Background (CMB) fluctuations from NASA's Wilkinson Microwave Anisotropy Probe (WMAP) [1] have been particularly spectacular, finding excellent agreement with independent cosmological observations and the general predictions of our inflationary $\Lambda \mathrm{CDM}$ cosmological model [2]. Specifically, the temperature perturbations are consistent with a Gaussian random field exhibiting a distinct power spectrum with peaks due to sound waves in the plasma before the epoch of recombination.

It is no surprise that the WMAP dataset has received a lot of attention, with various attempts to find and characterise anomalies that would signal a primordial departure from a Gaussian random field or contamination of the data (e.g. $3,44,5,6,7,6,5,10,11,12,13,14]$ ). While there are some indications of possible pathologies in the data, the statistical significances vary and the general consesus so far is that the data is not contaminated with foregrounds at a significant level.

However, a recent work has claimed that there are spatial associations between neutral hydrogen and smallscale CMB structure observed by WMAP [15]. These associations are claimed to be significant at scales of order $1^{\circ}$, which also corresponds to the scale of the first acoustic peak in the CMB power spectrum. This peak is an important "standard ruler" when it comes to parameter fitting and thus any such contamination could have serious consequences for the inferred power spectrum and cosmological parameter values. However, the statistical significance of these correlations are currently unclear. Visual inspection alone is insufficient as the human brain is particularly susceptible to discerning structure in random fields. After all, one can easily be convinced that Stephen Hawking's initials are imprinted in the WMAP Internal Linear Combination (ILC) map at $(l, b) \sim\left(60^{\circ}, 10^{\circ}\right)$.

In this paper we extend the work of [15] and perform a rigorous examination of the correlation between the third-year single-frequency WMAP maps and tracers of Hi structure from the Leiden/Argentine/Bonn Galactic Hi Survey (LAB) experiment [16]. There is no established mechanism by which the Galactic neutral hydrogen could affect the WMAP data, besides the fact that it spatially correlates with other contaminants. Thus, a statistically significant correlation would indicate that either there is a previously unknown emission process taking place or, more likely, that the CMB data has not been adequately cleaned or masked.

In this brief report we perform a series of tests, employing various sky-cuts and probing a range of angular scales. We find no statistically significant correlations. In Section $\amalg$ we present the datasets that we use, outline our cross-correlation method, and outline the process by which we will assess statistical significance. In Section III we present our results, while the last Section IV concludes the paper.

\section{DATA AND METHOD}

As a tracer of the neutral hydrogen we use data from the LAB survey [16]. The data is distributed in a 3dimensional cube spanning the full-sky with half-degree steps in galactic longitude and latitude. The third component traces the line of sight velocity $v$ spanning the interval $-450 \mathrm{~km} / \mathrm{s}$ to $+400 \mathrm{~km} / \mathrm{s}$. We have resampled the survey into a HEALPix [17 map with $N_{\text {side }}=512$. We tried several schemes of resampling and found that the results are independent of the exact scheme used. Results reported in this work correspond to the nearestneighbour resampling. We have prepared the following Hi maps:

- Full velocity (FV); An integral of all hydrogen data along the line of sight.

- High velocity (HV) ; Line of sight integral of $v<$ $-100 \mathrm{~km} / \mathrm{s}$.

- Intermediate velocity (IV) ; Line of sight integral of $-100 \mathrm{~km} / \mathrm{s}<v<-30 \mathrm{~km} / \mathrm{s}$. 

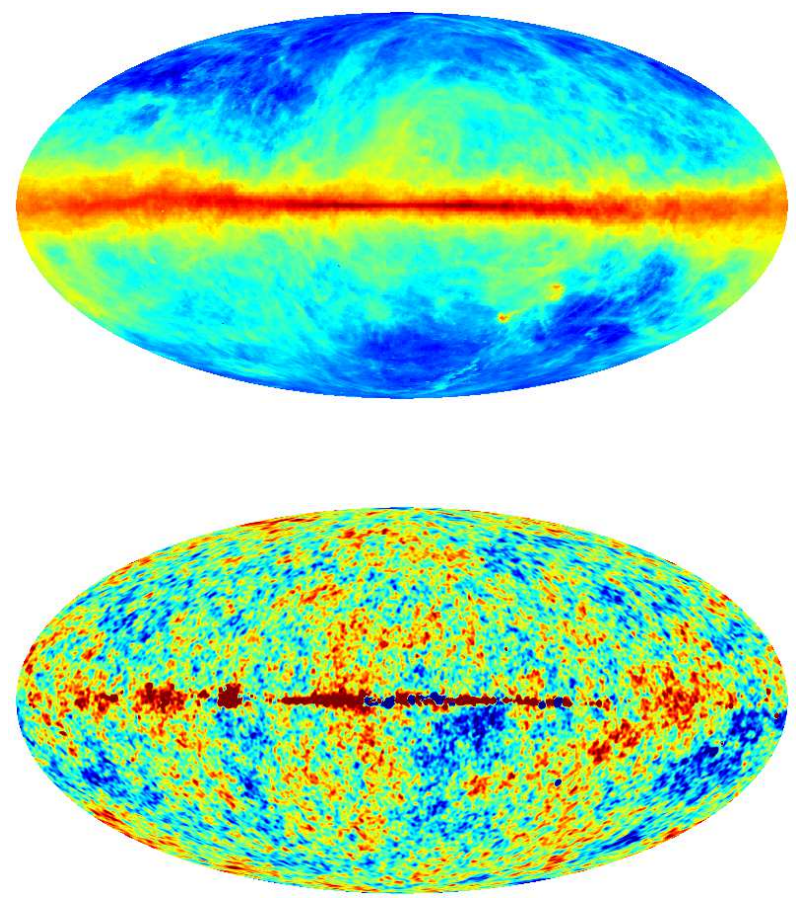

FIG. 1: The Full velocity LAB map (above) and the foreground reduced V-band WMAP map (below), in galactic coordinates and Mollweide projection. The scales are logarithmic in column density and linear in temperature fluctuation.

- Low velocity (LV) ; Line of sight integral of -30 $\mathrm{km} / \mathrm{s}<v<+30 \mathrm{~km} / \mathrm{s}$.

- Velocity cuts ; Various line of sight integrals over a $10 \mathrm{~km} / \mathrm{s}$ portion of the velocity range $-450 \mathrm{~km} / \mathrm{s}$ $<v<+400 \mathrm{~km} / \mathrm{s}$. Resulting in 85 maps, herein named according to their mean velocity, i.e. -445 , $-435, \ldots,+395 \mathrm{~km} / \mathrm{s}$.

The HV, IV, LV maps are defined to match those used by [15]. In Figure 1 we show the FV map.

For the CMB data we use the three-year WMAP foreground reduced temperature maps for the $\mathrm{Q}, \mathrm{V}$, and $\mathrm{W}$ frequency bands [18]. These were produced (by the WMAP collaboration) by subtracting synchrotron, freefree, and dust emission templates from the single year "unreduced" maps. Full three-year maps consist of single year maps coadded with inverse noise weighted coefficients, and the single frequency maps consist of the relevant differencing assembly data similarly coadded. In Figure 1 we show the V-band map (smoothed with a beam of $1^{\circ} \mathrm{FWHM}$ ).

The CMB data remains contaminated by galactic emission and a mask of the galactic plane is necessary. We employ three different masks.

- Kp2 ; The standard CMB mask, produced by the WMAP team (a 15.3\% cut)
- KpO ; A more aggressive intensity mask (a 23.5\% cut)

- Rectangle ; A mask exposing just the window $60^{\circ}<$ $l<180^{\circ}, 30^{\circ}<b<70^{\circ}$, as used in [15].

We perform the cross-correlation in harmonic space, over the range $\ell=2-200$, with the upper limit reflecting the resolution of LAB. The cross-correlation of the angular multipole $\ell$ is calculated according to

$$
X_{\ell}=\frac{1}{(2 \ell+1)} \sum_{m=-\ell}^{\ell} a_{\ell m}^{\mathrm{CMB}} a_{\ell m}^{* \mathrm{HI}},
$$

where the $a_{\ell m}$ are the spherical harmonic coefficients of the respective maps. The so-defined quantity is rotationally invariant and real. Assuming the datasets are Gaussian and uncorrelated the quantity $X_{\ell}$ will be normally distributed in the limit of large $\ell$ (by the central limit theorem), with corresponding standard deviation $\Delta X_{\ell}$ given by

$$
\left(\Delta X_{\ell}\right)^{2} \sim \frac{1}{f_{\text {sky }}(2 \ell+1)} C_{\ell}^{\mathrm{CMB}} C_{\ell}^{\mathrm{HI}},
$$

where $f_{\text {sky }}$ is the fraction of sky being observed. However, the LAB dataset is not expected to be Gaussian, and the presence of the mask correlates the maps somewhat. Therefore we determine the variance $\Delta X_{\ell}$ (and mean $\bar{X}_{\ell}$ ) by performing Monte-Carlo cross-correlations of the LAB maps with 2000 simulated WMAP maps, and we determine these statistics separately for all combinations of WMAP band, mask and LAB map. The WMAP simulations are created assuming the third-year WMAP only best-fit theoretical power spectrum, smoothed with the beam profiles of the relevant frequency band, and with noise added according to the $N_{\text {obs }}$ field in the individual maps. We assume the noise between pixels is uncorrelated; an approximation that holds very well for the WMAP temperature maps [1].

The effective $\chi^{2}$ is then calculated,

$$
\chi^{2}=\sum_{\ell=\ell_{\min }}^{\ell_{\max }}\left(\frac{X_{\ell}-\bar{X}_{\ell}}{\Delta X_{\ell}}\right)^{2} .
$$

Assuming independent multipoles this variable should be approximately $\chi^{2}$ distributed with $\left(\ell_{\max }-\ell_{\min }+1\right)$ degrees of freedom. However, to be more accurate we compare our resulting $\chi^{2}$ values to those from the simulations (for the same frequency band, LAB map, mask and $\ell$-range). Significance of the departure from a nullcorrelation is thus assessed through the percentage of simulations with lower $\chi^{2}$ values.

As a simple test, we have performed analysis with unmasked data and we observe a very large correlation that systematically varies with QVW band, as expected due to the neutral hydrogen tracing known contaminants such as dust, free-free, and synchrotron emission. 


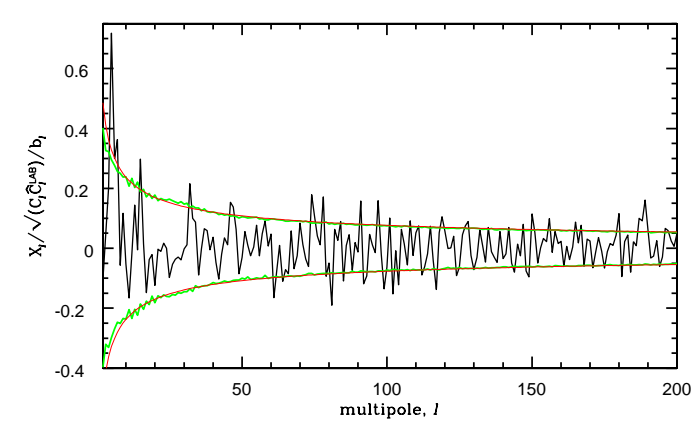

FIG. 2: The cross-correlation of the Full velocity LAB survey with WMAP V-band, outside the KP2 mask, divided by the power spectra and V-band beam. Also plotted are the 1-sigma contours calculated from 5000 simulations (green thick) and from Eqn(2) (red thin). The equivalent plot for the $\mathrm{Q}$ and W-bands are essentially identical to this one.

\section{RESULTS}

We consider 89 LAB maps in total, and 3 different masks. We further focus our attention on 3 different

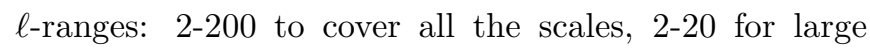
scales only, and 160-200 for near degree scales. We compute the $\chi^{2}$ values for all possible combinations of the 3 masks, 3 WMAP bands, 89 LAB maps, and $3 \ell$-ranges (using $\bar{X}_{\ell}, \Delta X_{\ell}$ values determined for the same maskband-map combination). In Figure 2 we plot an example cross-correlation result $X_{\ell}$, with 1-sigma contours returned from simulations and from Eqn (2).

In Table \we consider how many of our $\chi^{2}$ values are higher than $95 \%$ of those from simulations, and we find a total of 112 cross-correlation results high at this level. By definition, from a total of $89 \times 3 \times 3 \times 3=2403$ different $\chi^{2}$ numbers, we expect approximately 120 of our results to be significant at this level. Our results are therefore consistent with the null hypothesis of no correlation between the WMAP and LAB maps.

The number of significant results is binomially distributed, with the scatter depending on the number of independent results. Our 2403 results are definitely not independent: the $\mathrm{Q}, \mathrm{V}, \mathrm{W}$ results are almost identical (as can be deduced from the Table I and Figure 3), the $\ell$-ranges overlap, the masks are not exclusive, and the neighbouring velocity slices are highly correlated. This increases the expected spread relative to the mean, $(\sigma / \mu)^{2} \sim(1-p) /(N p)$, and thus from further Monte Carlo simulations we can determine the effective number of independent results that we have. From fitting a binomial distribution to a histogram of the number of significant (at the $95 \%$ level) $\chi^{2}$ results that 500 new simulations find, we observe an effective $N$ of $\sim 74$. We find a consistent result by fitting to the equivalent $5 \%$ confidence level results. We find $(\mu, \sigma) \sim(126,66)$, which provides further evidence that a total of 112 signficant values is consistent with no correlation.

We now turn to extreme values. The highest $\chi^{2}$ value was obtained for the correlation of $\mathrm{W}$-band $\mathrm{CMB}$ with

\begin{tabular}{|c|ccc|}
\hline & KP2 & KP0 & RECT \\
\hline $2-200$ & $3,5,4$ & $3,6,1$ & $2,2,1$ \\
$2-20$ & $1,1,1$ & $1,1,1$ & $0,0,0$ \\
$160-200$ & $10,15,8$ & $10,14,4$ & $5,9,4$ \\
\hline
\end{tabular}

TABLE I: The number of cross-correlations with $\chi^{2}$ value that is significant at the $95 \%$ level when compared to simulations. Numbers in each cell correspond to the Q,V and W-bands respectively.

a Hi slice at $215 \mathrm{~km} / \mathrm{s}$ (on degree scales, with the $\mathrm{Kp} 2$ mask), with only $0.07 \%$ of the $\mathrm{W}$-band simulations finding a higher such $\chi^{2}$ (we did a special run with $10^{4}$ simulated maps for this case). How unlikely is it to have such an extreme value given our sample size? If one draws $N$ numbers between 0 and 1 , the probability that they will all be less than $x$ is $p=x^{N}$. For $x=0.9993$ and $N=2403, p=0.19$, and therefore it appears entirely typical to get such an extreme value for this sample size if the results are independent. An improved calculation uses the effective number of independent results, determined to be $\sim 74$, for which we find $p=0.95$ - indicating that there is a $5 \%$ probability of obtaining such an extreme outlier. This result is very conservative, as in reality we have had more than 74 numbers from which to select our extreme value, benefitting from any extra variation. By considering limiting cases we see that the probability of our extrema lies somewhere between $5 \%$ and $81 \%$, and thus not unusal at any significant level (ie. above $3 \sigma$ ).

We note that in contrast with [15] we do not observe any systematic correlation between the HV, LV, IV, or FV HI maps and the CMB. Moreover, we do not observe a significant correlation between $\mathrm{HI}$ and the $\mathrm{CMB}$ in the area of the sky defined by the Rectangular mask, as just 2 of the 89 maps find some correlation above the $99 \%$ level, and for only one of the $\ell$-ranges - consistent with a chance occurence.

We do, however, observe some correlation on degree scales for a number of velocity slices, as seen in the third row of Table I. The 15 maps that demonstrate a Kp2 masked V-band degree scale correlation above the $95 \%$ level correspond to mean velocities of $-405,-345,-325$, $-315,-205,105 \ldots 155,215 \ldots 235,345 \mathrm{~km} / \mathrm{s}$. Since this is an a-posteriori observation and adjacent LAB slices are highly correlated, it is hard to ascertain the statistical significance of these. Nevertheless, we track the correlation of $105 \ldots 155 \mathrm{~km} / \mathrm{s}$ and $215 \ldots 235 \mathrm{~km} / \mathrm{s}$ maps to the same extended feature at $(l, b) \sim\left(-50^{\circ},-45^{\circ}\right)$ (none of the remaining 15 maps are significant above the $99 \%$ level). The fact that the correlation is due to just one feature again indicates how correlated the slices are, and effectively reduces the number of correlations in this case to just one. In Figure 3 we examine the correlation of 215 $\mathrm{km} / \mathrm{s}$ with the different frequency bands to ascertain if the signal is due to some kind of contamination from the presence of this extended Hi feature. However, the signal remains very consistent between the CMB bands indicat- 


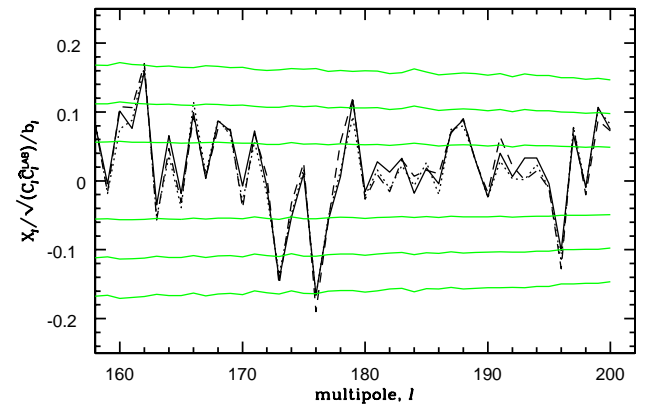

FIG. 3: The cross-correlation of the 215 Hi map with the WMAP data, outside the Kp2 mask, over scales $\ell=160-200$ where the $\chi^{2}$ is found to be high at the $99.93 \%$ level. To compare results from the different bands [Q(solid), V(dotted, $\mathrm{W}$ (dashed)] we have divided out by their repective beams. 1,2 and 3-sigma results are also displayed (green solid).

ing that there is no obvious foreground contamination on these scales. The correlation also appears quite random in nature, with no clear trend apparent in Figure 3 .

As discussed, some chance correlation between the LAB and WMAP data is expected and we do not observe any more than usual. Further, demonstrated by the low effective number of independent results (74), the velocity slices are highly correlated. Therefore correlations with one slice will inevitable lead to correlations with a set of neighboring velocity slices. Supported by the random nature of the signal in Figure 3 we conclude that this particular set of significant $\chi^{2}$ values does not demonstrate a deviation from the norm, but rather it is a manifestation of one of the inevitable chance correlations.

\section{CONCLUSIONS}

Correlations between Galactic HI and the CMB are not expected. Thus looking for such correlations can be a useful blind test of potential systematics, such as foreground contamination of the CMB maps. However, there are two important caveats.

Firstly, correlations will inevitably appear from random fluctuations and one must not use a-posteriori statistics to claim detections. Even if one is forced to do so, the required significance bar should be significantly higher than in the case of expected results. Secondly, correlations by eye are very misleading and quantitative methods must be employed. Today's Monte-Carlo methods allow for easy assessment of significance.

We tested for correlation between the third-year WMAP CMB maps and LAB data of Galactic HI. We considered three different masks, CMB frequency bands, angular scales and 89 different Hi velocity slices. We do not find any convincing evidence for a correlation. The lack of correlation demonstrates how impressively clean the WMAP CMB maps are, outside of the masked regions.

\section{Acknowledgements}

We thank the anonymous referee for their helpful suggestions, and taming the more cavalier parts of this report. KRL is funded by a Glasstone research fellowship and Christ Church college, AS by Oxford Astrophysics.
[1] G. Hinshaw et al. (WMAP) (2006), astro-ph/0603451.

[2] D. N. Spergel et al. (WMAP) (2006), astro-ph/0603449.

[3] E. Komatsu et al. (WMAP), Astrophys. J. Suppl. 148, 119 (2003), astro-ph/0302223.

[4] H. K. Eriksen, A. J. Banday, K. M. Gorski, F. K. Hansen, and P. B. Lilje, Astrophys. J. 660, L81 (2007), astro$\mathrm{ph} / 0701089$.

[5] K. Land and J. Magueijo, Mon. Not. Roy. Astron. Soc. 357, 994 (2005), astro-ph/0405519.

[6] A. Slosar and U. Seljak, Phys. Rev. D70, 083002 (2004), astro-ph/0404567.

[7] P. Vielva, E. Martinez-Gonzalez, R. B. Barreiro, J. L. Sanz, and L. Cayon, Astrophys. J. 609, 22 (2004), astro$\mathrm{ph} / 0310273$.

[8] X. Liu and S. N. Zhang, Astrophys. J. 633, 542 (2005), astro-ph/0504589.

[9] J. D. McEwen, M. P. Hobson, A. N. Lasenby, and D. J. Mortlock, Mon. Not. Roy. Astron. Soc. 359, 1583 (2005), astro-ph/0406604.
[10] J. D. McEwen, M. P. Hobson, A. N. Lasenby, and D. J. Mortlock, Mon. Not. Roy. Astron. Soc. Lett. 371, L50 (2006), astro-ph/0604305.

[11] H. K. Eriksen, D. I. Novikov, P. B. Lilje, A. J. Banday, and K. M. Gorski, Astrophys. J. 612, 64 (2004), astroph/0401276.

[12] A. Bernui, C. Tsallis, and T. Villela, Europhys. Lett. 78, 19001 (2007), astro-ph/0703708.

[13] L.-Y. Chiang, P. D. Naselsky, O. V. Verkhodanov, and M. J. Way, Astrophys. J. 590, L65 (2003), astro$\mathrm{ph} / 0303643$.

[14] K. M. Huffenberger, H. K. Eriksen, and F. K. Hansen, Astrophys. J. 651, L81 (2006), astro-ph/0606538.

[15] G. L. Verschuur (2007), arXiv:0704.1125 [astro-ph].

[16] P. M. W. Kalberla et al. (2005), astro-ph/0504140.

[17] K. M. Gorski et al., Astrophys. J. 622, 759 (2005), astroph/0409513.

[18] Available at http://lambda.gsfc.nasa.gov/ 